\title{
DIVERGENCES IN FORMAL VARIATIONAL CALCULUS AND BOUNDARY TERMS IN HAMILTONIAN FORMALISM
}

\author{
VLADIMIR O. SOLOVIEV \\ Institute for High Energy Physics \\ 142 284, Protvino, Moscow region, Russia \\ E-mail:vosoloviev@mx.ihep.su
}

\begin{abstract}
It is shown how to extend the formal variational calculus in order to incorporate integrals of divergences into it. Such a generalization permits to study nontrivial boundary problems in field theory on the base of canonical formalism.

1. Introduction. The Hamiltonian formulation of classical mechanics [Arn] is based on geometrical constructions which use such notions as differential forms, vector fields and multivectors, and such operations as differential, interior product, Lie derivative, Schouten-Nijenhuis bracket. Most of these constructions were extended to field theory in the process of studying nonlinear integrable models during the last 20 years [Olv86]. This approach has been called the formal variational calculus [GD] because it ignores any terms arising as a result of integration by parts. This is fully justified in case of periodic boundary conditions or fast decay of fields at spatial infinity, but unfortunately, this method is not applicable in its initial form to many other problems interesting from physical point of view. For example, massless fields are slowly decaying at infinity and, as a result, some important characteristics of these fields are expressed just through surface integrals (or volume integrals of spatial divergences). They are necessary to form canonical generators of the global gauge transformations or asymptotic symmetries of the Riemannian metric. The great efforts were started at the end of fifties to understand the role of surface terms in the Hamiltonian of General Relativity [ADM]. Only after 15 years of study the satisfactory explanation had been given [RT]. But even then not all questions were answered. For example, one might worry how to retain the surface terms which are necessary to realize the Poincaré algebra in asymptotically flat space [RT], [Sol85]

$$
\{H(\xi), H(\eta)\}=H([\xi, \eta])
$$
\end{abstract}

1991 Mathematics Subject Classification: Primary 58F05; Secondary 70G50, 58G20.

The paper is in final form and no version of it will be published elsewhere. 
when doing local calculations of the constraints algebra

$$
\begin{gathered}
\{\mathcal{H}(x), \mathcal{H}(y)\}=g^{a b}(x) \mathcal{H}_{b}(x) \delta_{, a}(x, y)-g^{a b}(y) \mathcal{H}_{b}(y) \delta_{, a}(y, x), \\
\left\{\mathcal{H}(x), \mathcal{H}_{a}(y)\right\}=-\mathcal{H}(y) \delta_{, a}(y, x), \\
\left\{\mathcal{H}_{a}(x), \mathcal{H}_{b}(y)\right\}=\mathcal{H}_{b}(x) \delta_{, a}(x, y)-\mathcal{H}_{a}(y) \delta_{, b}(y, x) .
\end{gathered}
$$

We will show in the following that all the main structures of the formal variational calculus can be extended on nontrivial contributions of divergences through introduction of a new grading and new pairing compatible with it. So, it occurs possible to preserve the nice geometrical language in the more general case than before. After all the field theory Poisson bracket is given by a new formula which differs from the standard one by surface terms. Simultaneously we get the answer to the mentioned problem of disappearance of the surface contributions in local calculations with $\delta$-function. The natural way to take the boundary terms into account is to introduce the characteristic function $\theta_{\Omega}(x)$ of the integration domain $\Omega$. Then relations like

$$
\left(\theta_{\Omega}(x) \frac{\partial}{\partial x^{i}}+\theta_{\Omega}(y) \frac{\partial}{\partial y^{i}}\right)=-\theta_{\Omega}^{(i)} \delta(x, y),
$$

give the solution. In its turn this is connected with the observation [Sol92] that transformations of the type (for example, transformation to Ashtekar's variables)

$$
q^{A}(x) \rightarrow q^{A}(x), \quad p_{A}(x) \rightarrow p_{A}(x)+\frac{\delta F[q]}{\delta q^{A}(x)},
$$

in field theory are canonical only up to boundary contributions, because the standard Euler-Lagrange variational derivatives in general do not commute [And76], [And78].

We expect that boundary conditions should be treated in this formalism as a kind of constraints put on the initial data, i.e., they should be added to the Hamiltonian with some Lagrange multipliers and then checked for compatibility with the dynamics. The requirement of compatibility may lead to secondary boundary conditions or to fixing the Lagrange multipliers. But now this subject is not enough studied and our consideration is preliminary and limited to one example: the nonlinear Schrödinger equation.

2. New Poisson bracket formula. Below we use the local coordinate language and instead of the manifold with a boundary consider a domain $\Omega$ in $\mathbb{R}^{n}$ having a smooth boundary $\partial \Omega$. We do not expect that global formulation could meet with serious difficulties.

Definition 1. An integral over a finite domain $\Omega$ of a function of field variables $\phi^{A}(x), A=1, \ldots, p$ and their partial derivatives $D_{J} \phi^{A}$ up to some finite order

$$
F=\int_{\Omega} d^{n} x f\left(\phi_{A}(x), D_{J} \phi_{A}(x)\right)
$$

is called a local functional.

Remark 1. In contrast to the standard definition we do not treat these local functionals as equivalent if they differ by a divergence term. 
All the functions $f$ and $\phi_{A}$ as well as their variations throughout the paper are supposed infinitely smooth, i.e. $C^{\infty}\left(\mathbb{R}^{n}\right)$. We use multi-index notations $J=\left(j_{1}, \ldots, j_{n}\right)$

$$
D_{J}=\frac{\partial^{|J|}}{\partial^{j_{1}} x^{1} \ldots \partial^{j_{n}} x^{n}}, \quad|J|=j_{1}+\ldots+j_{n}, \quad D_{0}=1 .
$$

The derivative operator $D$ will denote below the full partial derivative taking into account also coordinate dependence of fields $\phi_{A}(x)$. As the number of summations in some formulae of this paper is large we will write only a sign of summing without displaying the indices of summation. According to this rule, sum over all repeated indices should be understood. In those cases, where it is not so, we display the summation indices. Also, we do not show the limits of summation, because they are natural, i.e. outside them the summand is simply zero. Usually we omit $d^{n} x$ in the integrals and show the arguments only when they can be mixed.

We denote as $\mathcal{A}$ the space of local functionals. It is important that this space includes functionals with integrands depending on derivatives of arbitrary order [And92]. Otherwise the Poisson brackets could go out of $\mathcal{A}$. The following is the general definition of field theory Poisson bracket.

Definition 2. A bilinear operation $\{\cdot, \cdot\}$ such that for any $F, G, H \in \mathcal{A}$

1) $\{F, G\} \in \mathcal{A}$;

2) $\{F, G\}=-\{G, F\}$;

3) $\{\{F, G\}, H\}+\{\{H, F\}, G\}+\{\{G, H\}, F\}=0$;

is called the field theory Poisson bracket.

The key idea of the new formula is in exploitation of the full variations which are free on the boundary. The variation of a local functional

$$
\delta F=\sum \int \frac{\partial f}{\partial \phi_{A}^{(J)}} D_{J} \delta \phi_{A}
$$

contains the differential operator which is called Fréchet derivative

$$
f_{A}^{\prime}=\sum_{J=0}^{\infty} \frac{\partial f}{\partial \phi_{A}^{(J)}} D_{J}
$$

We propose to define field theory Poisson bracket by the formula

$$
\{F, G\}=\sum \int_{\Omega} \operatorname{Tr}\left(D_{f_{A}} \hat{I}_{A B} D_{g_{B}}\right),
$$

where trace of two differential operators is used

$$
\operatorname{Tr}(\hat{A} \hat{B})=\sum_{I, K} D_{I} A_{K} D_{K} B_{I},
$$

where

$$
\hat{A}=\sum_{K} A_{K} D_{K}, \quad \hat{B}=\sum_{I} B_{I} D_{I} .
$$

The important property of the trace is

$$
\operatorname{Tr}(\hat{A} D \hat{B})=D \operatorname{Tr}(\hat{A} \hat{B})=\operatorname{Tr}(D \hat{A} \hat{B}) .
$$


The individual structure of a Poisson bracket is given by matrix $I_{A B}$. More general treatment of it will be given in the next section and here it is simply constant and antisymmetric matrix.

Symmetrized covariant derivatives can also be used in the expression for the first variation of local functional. We can replace partial derivatives by covariant ones in the trace calculation if the curvature is zero or if one operator is simply a function. Then covariance of the new formula under changes of independent variables is evident. In general case a special consideration is necessary.

The new bracket differs from the standard one in the exact fulfilment of the Jacobi identity under arbitrary boundary conditions [Sol93]. In the same time its calculation is not more complicated than usual since we need not integrate by parts to get EulerLagrange derivatives.

We can also use another representation of the first variation [Olv86]

$$
\delta F=\sum \int_{\Omega} D_{J}\left(E_{A}^{J}(f) \delta \phi_{A}\right) .
$$

where the higher Eulerian operators [KMGZ], [Ald]

$$
E_{A}^{J}(f)=\sum_{K}(-1)^{|K|+|J|}\left(\begin{array}{c}
K \\
J
\end{array}\right) D_{K-J} \frac{\partial f}{\partial \phi_{A}^{(K)}},
$$

are used. The zero order operator is just the standard Euler-Lagrange variational derivative. Binomial coefficients for multi-indices are

$$
\left(\begin{array}{c}
J \\
K
\end{array}\right)=\left(\begin{array}{l}
j_{1} \\
k_{1}
\end{array}\right) \ldots\left(\begin{array}{l}
j_{n} \\
k_{n}
\end{array}\right)
$$

where ordinary binomial coefficients are

$$
\left(\begin{array}{l}
j \\
k
\end{array}\right)= \begin{cases}j ! /(k !(j-k) !) & \text { if } 0 \leq k \leq j \\
0 & \text { otherwise }\end{cases}
$$

Let us mention that if $J$ is not contained in $K$, then all quantities having multi-index $(K-J)$ are zero. The sums over $J$ and $K$ above are really finite because local functional can depend only on a finite number of derivatives according to Definition 1.

A remarkable property of these operators is

$$
E_{A}^{J}\left(D_{I} f\right)=E_{A}^{J-I}(f) .
$$

It leads to the result that if

$$
\left\{\int f, \int g\right\}=\int h
$$

then

$$
\left\{\int D f, \int g\right\}=\left\{\int f, \int D g\right\}=\int D h .
$$

3. Extension of the formal variational calculus. In dealing with terms arising in the integration by parts it is suitable to represent integrals over finite domain as integrals over infinite space with the help of the characteristic function

$$
\theta_{\Omega}(x)= \begin{cases}1 & \text { if } x \in \Omega \\ 0 & \text { otherwise }\end{cases}
$$


We can understand it also as Heaviside function $\theta_{\Omega}(x)=\theta\left(P_{\Omega}\right)$, where equation $P_{\Omega}(x)=$ 0 defines the boundary and

$$
P_{\Omega}(x) \begin{cases}>0 & \text { if } x \in \Omega \\ =0 & \text { if } x \in \partial \Omega \\ <0 & \text { otherwise. }\end{cases}
$$

Then

$$
F=\int_{\Omega} f=\int \theta_{\Omega} f
$$

and, for example, the full variation of a local functional can be expressed in the form

$$
\delta F=\int \frac{\delta F}{\delta \phi_{A}} \delta \phi_{A}
$$

where the distribution

$$
\frac{\delta F}{\delta \phi_{A}}=E_{A}^{0}\left(\theta_{\Omega} f\right)=\sum(-1)^{|J|} D_{J} \theta_{\Omega} E_{A}^{J}(f)
$$

could be called the full variational derivative. Such a representation corresponds to the situation opposite to the standard: here distributions are of finite support whereas test functions $\delta \phi_{A}$ are arbitrary.

A grading in linear space $L$ is a decomposition of it into direct sum of subspaces, with a special value of some function $p$ (grading function) assigned to all the elements of any subspace [Dorf]. Elements of each subspace are called homogeneous.

In our case the factor $D_{J} \theta_{\Omega}$ is responsible for the grading and the function $p$ takes its values in the set of all positive multi-indices $J=\left(j_{1}, \ldots, j_{n}\right)$

$$
L=\bigoplus_{J=0}^{\infty} L^{\langle J\rangle}
$$

We always can return to the standard formal variational calculus by putting $\theta_{\Omega}(x) \equiv 1$.

A bilinear operation $x, y \mapsto x \circ y$, defined on $L$, is said to be compatible with the grading if the product of any homogeneous elements is also homogeneous, and if

$$
p(x \circ y)=p(x)+p(y) .
$$

3.1. Local functionals and evolutionary vector fields. Here we will call the expression given in Definition 1 the canonical form of a local functional. We formally extend that definition by allowing local functionals to be written as follows

$$
F=\sum_{J=0}^{\infty} \int D_{J} \theta_{\Omega}(x) f^{\langle J\rangle}\left(\phi_{A}(x), D_{K} \phi_{A}(x)\right) d^{n} x=\sum \int \theta^{(J)} f^{\langle J\rangle},
$$

where in accordance with the previous definition only a finite number of terms is allowed. Here and below we simplify the notation for derivatives of $\theta$ and remove $\Omega$. Of course, any functional of such a form can be transformed to the form used above through integration by parts with

$$
f=\sum(-1)^{|J|} D_{J} f^{\langle J\rangle}
$$

So, the formal integration by parts over infinite space $\mathbb{R}^{n}$ evidently changes the grading. It will be clear below that the general situation is from one side compatibility of all 
bilinear operations with the grading and from the other side with formal integration by parts. So, basic objects (local functionals etc.) are defined as equivalence classes modulo formal divergences (i.e., divergences of expressions containing $\theta$-factors) and the unique decomposition into homogeneous subspaces with fixed grading function can be made only for representatives of these classes. But we will see that the pairing will be defined in such a way to avoid any ambiguity.

We call expressions of the form

$$
\psi=\sum \int \theta^{(J)} D_{K} \psi_{A}^{\langle J\rangle} \frac{\partial}{\partial \phi_{A}^{(K)}}
$$

the evolutionary vector fields. The expressions $\psi_{A}^{\langle J\rangle}$ are called characteristics of them. The value of the evolutionary vector field on a local functional is given by the expression

$$
\psi F=\sum \int \theta^{(I+J)} D_{K} \psi_{A}^{\langle J\rangle} \frac{\partial f^{\langle I\rangle}}{\partial \phi_{A}^{(K)}} .
$$

It is a straightforward calculation to check that this operation is compatible with the formal integration by parts, i.e.

$$
\psi \operatorname{Div}(f)=\operatorname{Div}(\psi f),
$$

as it is in the standard formal variational calculus. This relation is, of course, valid for integrands.

It is easy to check that the evolutionary vector field with coefficients

$$
\psi_{A}^{(J)}=\sum\left(D_{L} \xi_{B}^{\langle I\rangle} \frac{\partial \eta_{A}^{\langle J-I\rangle}}{\partial \phi_{B}^{(L)}}-D_{L} \eta_{B}^{\langle I\rangle} \frac{\partial \xi_{A}^{\langle J-I\rangle}}{\partial \phi_{B}^{(L)}}\right)
$$

can be considered as the commutator of the evolutionary vector fields $\xi$ and $\eta$

$$
\psi F=[\xi, \eta] F=\xi(\eta F)-\eta(\xi F),
$$

with the Jacobi identity fulfilled for the commutator operation. Therefore the vector fields form a Lie algebra.

3.2. Differentials and functional forms. The differential of a local functional is simply the first variation of it

$$
\mathrm{d} F=\sum \int \theta^{(J)} \frac{\partial f^{\langle J\rangle}}{\partial \phi_{A}^{(K)}} \delta \phi_{A}^{(K)},
$$

here and below $\delta \phi_{A}^{(K)}=D_{K} \delta \phi_{A}$. It can also be expressed through the Fréchet derivative or through the higher Eulerian operators

$$
\mathrm{d} F=\sum \int \theta^{(J)} f^{\langle J\rangle^{\prime}}(\delta \phi)=\sum \int \theta^{(J)} D_{K}\left(E_{A}^{K}\left(f^{\langle J\rangle}\right) \delta \phi_{A}\right)
$$

This differential is a special example of functional 1-form. A general functional 1-form can be written as

$$
\alpha=\sum \int \theta^{(J)} \alpha_{A K}^{\langle J\rangle} \delta \phi_{A}^{(K)}
$$

Of course, the coefficients are not unique since we can do formal integration by parts. 
Let us call the following expression the canonical form of functional 1-form

$$
\alpha=\sum \int \theta^{(J)} \alpha_{A}^{\langle J\rangle} \delta \phi_{A}
$$

Analogously, we can define functional $m$-forms as integrals or equivalence classes modulo formal divergences of vertical forms

$$
\alpha=\frac{1}{m !} \sum \int \theta^{(J)} \alpha_{A_{1} K_{1}, \ldots, A_{m} K_{m}}^{\langle J\rangle} \delta \phi_{A_{1}}^{\left(K_{1}\right)} \wedge \ldots \wedge \delta \phi_{A_{m}}^{\left(K_{m}\right)}
$$

Define the pairing of an evolutionary vector field and 1-form as

$$
\alpha(\xi)=\xi\lrcorner \alpha=\sum \int \theta^{(I+J)} \alpha_{A K}^{\langle J\rangle} D_{K} \xi_{A}^{\langle I\rangle} .
$$

The interior product of an evolutionary vector field and functional $m$-form will be

$$
\begin{gathered}
\xi\lrcorner \alpha=\frac{1}{m !} \sum(-1)^{i+1} \int \theta^{(J+I)} \alpha_{A_{1} K_{1}, \ldots, A_{m} K_{m}}^{\langle J\rangle} D_{K_{i}} \xi_{A_{i}}^{\langle I\rangle} \delta \phi_{A_{1}}^{\left(K_{1}\right)} \wedge \ldots \\
\ldots \wedge \delta \phi_{A_{i-1}}^{\left(K_{i-1}\right)} \wedge \delta \phi_{A_{i+1}}^{\left(K_{i+1}\right)} \wedge \ldots \wedge \delta \phi_{A_{m}}^{\left(K_{m}\right)} .
\end{gathered}
$$

Then the value of $m$-form on the $m$ evolutionary vector fields will be defined by formula

$$
\left.\left.\alpha\left(\xi_{1}, \ldots, \xi_{m}\right)=\xi_{m}\right\lrcorner \ldots \xi_{1}\right\lrcorner \alpha .
$$

It can be checked by straightforward calculation that

$$
\operatorname{Div}(\alpha)\left(\xi_{1}, \ldots, \xi_{m}\right)=\operatorname{Div}\left(\alpha\left(\xi_{1}, \ldots, \xi_{m}\right)\right) .
$$

The differential of $m$-form given as

$$
\mathrm{d} \alpha=\frac{1}{m !} \sum \int \theta^{(J)} \frac{\partial \alpha_{A_{1} K_{1}, \ldots, A_{m} K_{m}}^{\langle J\rangle}}{\partial \phi_{A}^{(K)}} \delta \phi_{A}^{(K)} \wedge \delta \phi_{A_{1}}^{\left(K_{1}\right)} \wedge \ldots \wedge \delta \phi_{A_{m}}^{\left(K_{m}\right)},
$$

satisfies standard properties

$$
\mathrm{d}^{2}=0
$$

and

$$
\begin{aligned}
\mathrm{d} \alpha\left(\xi_{1}, \ldots, \xi_{m+1}\right)=\sum_{i}(-1)^{i+1} \xi_{i} \alpha\left(\xi_{1}, \ldots, \hat{\xi}_{i}, \ldots, \xi_{m+1}\right)+ & \\
& +\sum_{i<j}(-1)^{i+j} \alpha\left(\left[\xi_{i}, \xi_{j}\right], \xi_{1}, \ldots, \hat{\xi}_{i}, \ldots, \hat{\xi}_{j}, \ldots, \xi_{m+1}\right) .
\end{aligned}
$$

The Lie derivative of a functional form $\alpha$ along an evolutionary vector field $\xi$ can be introduced by the standard formula

$$
\left.\left.L_{\xi} \alpha=\xi\right\lrcorner \mathrm{d} \alpha+\mathrm{d}(\xi\lrcorner \alpha\right) .
$$

3.3. Graded differential operators and their adjoints. We call linear differential operators of the form

$$
\hat{I}=\sum_{J=0}^{\infty} \theta^{(J)} \sum_{N=0}^{N_{\max }} I_{A B}^{\langle J\rangle N} D_{N}
$$

graded differential operators. 
Let us call linear differential operator $\hat{I}^{*}$ adjoint to $\hat{I}$ if for arbitrary set of smooth functions $f_{A}, g_{A}$

$$
\sum_{A, B} \int f_{A} \hat{I}_{A B} g_{B}=\sum_{A, B} \int g_{A} \hat{I}_{A B}^{*} f_{B} .
$$

For coefficients of the adjoint operator we can derive the expression

$$
I_{A B}^{*\langle J\rangle M}=\sum_{K=0}^{K_{\max }} \sum_{L=0}^{\min (K, J)}(-1)^{|K|}\left(\begin{array}{c}
K \\
L
\end{array}\right)\left(\begin{array}{c}
K-L \\
M
\end{array}\right) D_{K-L-M} I_{B A}^{\langle J-L\rangle K} .
$$

It is easy to check that the relation

$$
\hat{I}(x) \delta(x, y)=\hat{I}^{*}(y) \delta(x, y)
$$

is valid. For example, we have

$$
\left(\theta(x) \frac{\partial}{\partial x^{i}}+\theta(y) \frac{\partial}{\partial y^{i}}\right)=-\theta^{(i)} \delta(x, y) .
$$

Operators satisfying relation

$$
\hat{I}^{*}=-\hat{I}
$$

will be called skew-adjoint. With the help of them it is possible to express 2-forms (and also 2 -vectors to be defined below) in the canonical form

$$
\alpha=\frac{1}{2} \sum_{A, B} \int \delta \phi_{A} \wedge \hat{I}_{A B} \delta \phi_{B} .
$$

It is clear that we can consider these representations of functional forms as formal decompositions over the basis derived as result of the tensor product of $\delta \phi_{A}$, with the totally antisymmetric multilinear operators

$$
\hat{\alpha}=\sum \theta^{(J)} \alpha_{A_{1} K_{1}, \ldots, A_{m} K_{m}}^{\langle J\rangle}\left(D_{K_{1}} \cdot, \ldots, D_{K_{m}} \cdot\right)
$$

being coefficients of these decompositions.

3.4. Multi-vectors and Schouten-Nijenhuis bracket. Let us introduce the dual basis to $\left|\delta \phi_{A}\right\rangle$ by formal relation

$$
\left\langle\frac{\delta}{\delta \phi_{B}(y)}, \delta \phi_{A}(x)\right\rangle=\delta_{A B} \delta(x, y),
$$

and construct by means of the tensor product a basis

$$
\frac{\delta}{\delta \phi_{B_{1}}(y)} \otimes \frac{\delta}{\delta \phi_{B_{2}}(y)} \otimes \ldots \otimes \frac{\delta}{\delta \phi_{B_{m}}(y)} .
$$

Then by using totally antisymmetric multilinear operators we can define functional m-vectors (or multi-vectors)

$$
\psi=\frac{1}{m !} \sum \int \theta^{(J)} \psi_{B_{1} L_{1}, \ldots, B_{m} L_{m}}^{\langle J\rangle} D_{L_{1}} \frac{\delta}{\delta \phi_{B_{1}}} \wedge \ldots \wedge D_{L_{m}} \frac{\delta}{\delta \phi_{B_{m}}} .
$$

Here a natural question arises: what is the relation between evolutionary vector fields and 1-vectors? Evidently, evolutionary vector fields lose their form when integrated by parts whereas 1-vectors conserve it. It is possible to prove the following Proposition [Sol94]. 
Proposition 1. There is a one-to-one correspondence between evolutionary vector fields and functional 1-vectors. The coefficients of 1-vector in the canonical form $\xi_{A}^{\langle J\rangle}$ are equal to the characteristic of the evolutionary vector field.

It is not difficult to show that we can define pairing (interior product) of 1-forms and 1 -vectors and this pairing preserves the identification

$$
\alpha(\xi)=\sum \int \theta^{(I+J)} \operatorname{Tr}\left(\alpha^{\langle I\rangle} \xi^{\langle J\rangle}\right) .
$$

When 1-vector is in the canonical form this result coincides with (1).

The interior product of 1-vector and $m$-form or, analogously, of 1-form and $m$-vector is defined as

$$
\begin{aligned}
\xi\lrcorner \alpha=\frac{1}{m !} \sum(-1)^{(i+1)} \int \theta^{(I+J)} D_{K_{i}} \xi_{A_{i} L}^{\langle I\rangle} D_{L}\left(\alpha_{A_{1} K_{1}, \ldots, A_{m} K_{m}}^{\langle J\rangle} \delta \phi_{A_{1}}^{\left(K_{1}\right)} \wedge \ldots\right. \\
\left.\ldots \wedge \delta \phi_{A_{i-1}}^{\left(K_{i-1}\right)} \wedge \delta \phi_{A_{i+1}}^{\left(K_{i+1}\right)} \wedge \ldots \wedge \delta \phi_{A_{m}}^{\left(K_{m}\right)}\right) .
\end{aligned}
$$

Then we can also define the value of $m$-form on $m$ 1-vectors (or, analogously, $m$-vector on $m$ 1-forms)

$$
\left.\left.\alpha\left(\xi_{1}, \ldots, \xi_{m}\right)=\xi_{m}\right\lrcorner \ldots \xi_{1}\right\lrcorner \alpha=\sum \int \theta^{\left(J+I_{1}+\ldots+I_{m}\right)} \operatorname{Tr}\left(\alpha^{\langle J\rangle} \xi_{1}^{\left\langle I_{1}\right\rangle} \ldots \xi_{m}^{\left\langle I_{m}\right\rangle}\right),
$$

where in this trace each entry of multilinear operator $\alpha$ acts only to the one corresponding $\xi$, whereas each derivation of the operator $\xi$ acts on the product of $\alpha$ and all the rest of $\xi$ 's.

It is possible to extend the differential onto $m$-vectors

$$
\mathrm{d} \psi=\frac{1}{m !} \sum \int \theta^{(J)} \frac{\partial \psi_{A_{1} K_{1}, \ldots, A_{m} K_{m}}^{\langle J\rangle}}{\partial \phi_{B}^{(L)}} \delta \phi_{B}^{(L)} D_{K_{1}} \frac{\delta}{\delta \phi_{A_{1}}} \wedge \ldots \wedge D_{K_{m}} \frac{\delta}{\delta \phi_{A_{m}}},
$$

and analogously onto mixed objects. Evidently, $\mathrm{d}^{2} \psi=0$.

With the help of the previous constructions we can define the Schouten-Nijenhuis bracket as follows

$$
\left.\left.[\xi, \eta]_{S N}=\mathrm{d} \xi\right\lrcorner \eta+(-1)^{p q} \mathrm{~d} \eta\right\lrcorner \xi
$$

for two multi-vectors of orders $p$ and $q$. The result of this operation is $p+q-1$-vector and it is analogous to the Schouten-Nijenhuis bracket in tensor analysis [Nij]. Its use in formal variational calculus is described in [Dorf]. However this bracket is defined only for operators there. We can recommend [Olv84] as an interesting source for treatment of the Schouten-Nijenhuis bracket for functional multi-vectors. Our construction of this bracket guarantees compatibility with the equivalence modulo divergences

$$
[\operatorname{Div}(\xi), \eta]_{S N}=\operatorname{Div}[\xi, \eta]_{S N}=[\xi, \operatorname{Div}(\eta)]_{S N} .
$$

Proposition 2. The Schouten-Nijenhuis bracket of functional 1-vectors up to a sign coincides with the commutator of corresponding evolutionary vector fields.

Pro of. Let us take two 1-vectors in canonical form

$$
\xi=\sum \int \theta^{(J)} \xi_{A}^{\langle J\rangle} \frac{\delta}{\delta \phi_{A}}, \quad \eta=\sum \int \theta^{(K)} \eta_{B}^{\langle K\rangle} \frac{\delta}{\delta \phi_{B}},
$$


and compute

$$
\left.\left.[\xi, \eta]_{S N}=\mathrm{d} \xi\right\lrcorner \eta-\mathrm{d} \eta\right\lrcorner \xi
$$

We have

$$
\mathrm{d} \xi=\sum \int \theta^{(J)} \xi_{A}^{\langle J\rangle^{\prime}}(\delta \phi) \frac{\delta}{\delta \phi_{A}}=\sum \int \theta^{(J)} \frac{\partial \xi_{A}^{\langle J\rangle}}{\partial \phi_{C}^{(L)}} \delta \phi_{C}^{(L)} \frac{\delta}{\delta \phi_{A}}
$$

and

$$
\mathrm{d} \xi\lrcorner \eta=-\sum \int \theta^{(J+K)} \frac{\partial \xi_{A}^{\langle J\rangle}}{\partial \phi_{B}^{(L)}} D_{L} \eta_{B}^{\langle K\rangle} \frac{\delta}{\delta \phi_{A}} .
$$

Therefore, we obtain

$$
[\xi, \eta]_{S N}=-\sum \int \theta^{(J+K)}\left(D_{L} \eta_{B}^{\langle K\rangle} \frac{\partial \xi_{A}^{\langle J\rangle}}{\partial \phi_{B}^{(L)}}-D_{L} \xi_{B}^{\langle K\rangle} \frac{\partial \eta_{A}^{\langle J\rangle}}{\partial \phi_{B}^{(L)}}\right) \frac{\delta}{\delta \phi_{A}}=-[\xi, \eta]
$$

Proposition 3 (Olver's Lemma [Olv86]). The Schouten-Nijenhuis bracket of the two bivectors can be expressed in the form

$$
[\xi, \psi]_{S N}=-\frac{1}{2} \sum \int \xi \wedge \hat{I}^{\prime}(\hat{K} \xi) \wedge \xi-\frac{1}{2} \sum \int \xi \wedge \hat{K}^{\prime}(\hat{I} \xi) \wedge \xi
$$

where the two differential operators $\hat{I}, \hat{K}$ are the coefficients of the bivectors in their canonical form.

Proof. Let us consider the Schouten-Nijenhuis bracket for the two bivectors and without loss of generality take them in the canonical form

$$
\begin{aligned}
& \chi=\frac{1}{2} \sum \int \theta^{(L)} \xi_{A} \wedge I_{A B}^{\langle L\rangle N} D_{N} \xi_{B}, \\
& \psi=\frac{1}{2} \sum \int \theta^{(M)} \xi_{C} \wedge K_{C D}^{\langle M\rangle P} D_{P} \xi_{D},
\end{aligned}
$$

where $\xi_{A}=\delta / \delta \phi_{A}$ and operators $\hat{I}, \hat{K}$ are skew-adjoint. Then we have

$$
\mathrm{d} \chi=\frac{1}{2} \sum \int \theta^{(L)} \frac{\partial I_{A B}^{\langle L\rangle N}}{\partial \phi_{E}^{(J)}} \delta \phi_{E}^{(J)} \xi_{A} \wedge D_{N} \xi_{B}
$$

and

$$
\begin{aligned}
\mathrm{d} \chi\lrcorner \psi=\frac{1}{4} \sum \int & \theta^{(L+M)} \frac{\partial I_{A B}^{\langle L\rangle N}}{\partial \phi_{C}^{(J)}} D_{J}\left(K_{C D}^{\langle M\rangle P} D_{P} \xi_{D}\right) \wedge \xi_{A} \wedge D_{N} \xi_{B}- \\
& -\frac{1}{4} \sum \int \theta^{(L+M)} D_{P}\left(\frac{\partial I_{A B}^{(L) N}}{\partial \phi_{D}^{(J)}} \xi_{A} \wedge D_{N} \xi_{B}\right) \wedge D_{J}\left(\xi_{C} K_{C D}^{\langle M\rangle P}\right) .
\end{aligned}
$$

Now let us make integration by parts in the second term

$$
\begin{aligned}
\mathrm{d} \chi & \lrcorner \psi=-\frac{1}{4} \sum \int \theta^{(L+M)} \xi_{A} \wedge\left(I_{A B}^{\langle L\rangle N}\right)^{\prime}\left(\hat{K}^{\langle M\rangle} \xi\right) \wedge D_{N} \xi_{B}- \\
- & \frac{1}{4} \sum \int \theta^{(L+M+Q)}(-1)^{|P|}\left(\begin{array}{c}
P \\
Q
\end{array}\right) \frac{\partial I_{A B}^{\langle L\rangle N}}{\partial \phi_{D}^{(J)}} \xi_{A} \wedge D_{N} \xi_{B} \wedge D_{J+P-Q}\left(\xi_{C} K_{C D}^{\langle M\rangle P}\right) .
\end{aligned}
$$


At last we change the order of multipliers under wedge product in the second term, make a replacement $M \rightarrow M-Q$ and organize the whole expression in the form

$$
\begin{aligned}
\mathrm{d} \chi\lrcorner \psi=-\frac{1}{4} \sum & \int \theta^{(L+M)} \xi_{A} \wedge\left(I_{A B}^{\langle L\rangle N}\right)_{C}^{\prime}\left(\hat{K}_{C D}^{\langle M\rangle} \xi_{D}+\right. \\
& \left.+(-1)^{|P|}\left(\begin{array}{c}
P \\
Q
\end{array}\right)\left(\begin{array}{c}
P-Q \\
R
\end{array}\right) D_{P-Q-R} K_{C D}^{\langle M-Q\rangle P} D_{R} \xi_{C}\right) \wedge D_{N} \xi_{B} .
\end{aligned}
$$

Having in mind the definition of adjoint operator (2) we can represent the final result of the calculation as follows,

$$
[\xi, \psi]_{S N}=-\frac{1}{2} \sum \int \theta^{(L+M)} \xi \wedge\left(\left(\hat{I}^{\langle L\rangle}\right)^{\prime}\left(\hat{K}^{\langle M\rangle} \xi\right)+\left(\hat{K}^{\langle M\rangle}\right)^{\prime}\left(\hat{I}^{\langle L\rangle} \xi\right)\right) \wedge \xi,
$$

therefore supporting in this extended formulation the method, proposed in [Olv86] for testing the Jacobi identity.

3.5. Poisson brackets and Hamiltonian vector fields. Let us call a bivector

$$
\Psi=\frac{1}{2} \sum \int \frac{\delta}{\delta \phi_{A}} \wedge \hat{I}_{A B} \frac{\delta}{\delta \phi_{B}},
$$

formed with the help of the graded skew-adjoint differential operator

$$
\hat{I}_{A B}=\sum \theta^{(L)} I_{A B}^{\langle L\rangle N} D_{N},
$$

the Poisson bivector if

$$
[\Psi, \Psi]_{S N}=0 .
$$

The operator $\hat{I}_{A B}$ is then called the Hamiltonian operator.

We may call the value of the Poisson bivector on differentials of the two functionals $F, G$

$$
\{F, G\}=\Psi(\mathrm{d} F, \mathrm{~d} G)=\mathrm{d} G\lrcorner \mathrm{d} F\lrcorner \Psi
$$

the Poisson bracket of these functionals.

The explicit form of the Poisson brackets can easily be obtained. It depends on the explicit form of the differential of the functionals, which can be changed by partial integration. Of course, all the possible forms are equivalent. Taking the extreme cases we have the expression through Fréchet derivatives

$$
\{F, G\}=\sum \int \theta^{(J)} \operatorname{Tr}\left(f_{A}^{\prime} \hat{I}_{A B}^{\langle J\rangle} g_{B}^{\prime}\right)
$$

or through higher Eulerian operators

$$
\{F, G\}=\sum \int \theta^{(J)} D_{P+Q}\left(E_{A}^{P}(f) \hat{I}_{A B}^{\langle J\rangle} E_{B}^{Q}(g)\right) .
$$

Theorem 1. The Poisson bracket defined above satisfies Definition 1.

Proof. It follows from the three facts:

1) from the previous formulas (3), (4) it is clear that $\{F, G\}$ is a local functional,

2) antisymmetry of $\{F, G\}$ is evident and

3) equivalence of the Jacobi identity to the Poisson bivector property can be proved [Sol94]. 
The result of interior product of the differential of a local functional $H$ and the Poisson bivector (up to the sign) will be called the Hamiltonian vector field (or the Hamiltonian 1-vector)

$$
\hat{I} \mathrm{~d} H=-\mathrm{d} H\lrcorner \Psi
$$

corresponding to the Hamiltonian $H$.

Evidently, the standard relations take place

$$
\{F, H\}=\mathrm{d} F(\hat{I} \mathrm{~d} H)=(\hat{I} \mathrm{~d} H) F .
$$

TheOREM 2. The Hamiltonian vector field corresponding to Poisson bracket of the functionals $F$ and $H$ coincides up to the sign with commutator of the Hamiltonian vector fields corresponding to these functionals.

Proof. Consider the value of commutator of Hamiltonian vector fields $\hat{I} \mathrm{~d} F$ and $\hat{I} \mathrm{~d} H$ on arbitrary functional $G$

$$
\begin{aligned}
& {[\hat{I} \mathrm{~d} F, \hat{I} \mathrm{~d} H] G=\hat{I} \mathrm{~d} F(\hat{I} \mathrm{~d} H(G))-\hat{I} \mathrm{~d} H(\hat{I} \mathrm{~d} F(G))=} \\
& =\hat{I} \mathrm{~d} F(\{G, H\})-\hat{I} \mathrm{~d} H(\{G, F\})=\{\{G, H\}, F\}-\{\{G, F\}, H\}= \\
& =-\{G,\{F, H\}\}=-\hat{I} \mathrm{~d}\{F, H\}(G),
\end{aligned}
$$

where we have used the Jacobi identity and antisymmetry of Poisson bracket. Due to arbitrariness of $G$ the proof is completed.

EXAMPLE 1. Let us consider a first structure

$$
\{u(x), u(y)\}=D_{x} \delta(x, y)
$$

of the Korteweg-de Vries equation ([Olv86], Example 7.6)

$$
u_{t}=u_{x x x}+u u_{x}
$$

Construct the adjoint graded operator to $\theta D$ according to (2)

$$
(\theta D)^{*}=-\theta D-D \theta
$$

and the skew-adjoint operator is

$$
\hat{I}=\frac{1}{2}\left(\theta D-(\theta D)^{*}\right)=\theta D+\frac{1}{2} D \theta .
$$

The Poisson bivector has the form

$$
\Psi=\frac{1}{2} \int \theta\left(\frac{\delta}{\delta u} \wedge D \frac{\delta}{\delta u}\right) .
$$

Differential of a local functional $H$ (for simplicity we suppose it is written in canonical

$$
H=\int \theta h
$$

form) is equal to

$$
\mathrm{d} H=\int \theta h^{\prime}(\delta u)=\sum_{k=0}^{\infty} \int \theta^{(k)}(-1)^{k} E^{k}(h) \delta u,
$$


where Fréchet derivative or higher Eulerian operators can be used. Therefore, the Hamiltonian vector field generated by $H$ is

or

$$
\hat{I} \mathrm{~d} H=-\mathrm{d} H\lrcorner \Psi=-\frac{1}{2} \int \theta\left[h^{\prime}\left(D \frac{\delta}{\delta u}\right)-D h^{\prime}\left(\frac{\delta}{\delta u}\right)\right],
$$

$$
-\frac{1}{2} \int \theta^{(k)}(-1)^{k}\left[E^{k}(h) D-D E^{k}(h)\right] \frac{\delta}{\delta u},
$$

or also

$$
-\frac{1}{2} \int \theta^{(k)}(-1)^{k} D_{i}\left[E^{k}(h) D-D E^{k}(h)\right] \frac{\partial}{\partial u^{(i)}} .
$$

The value of this vector field on another functional $F$ coincides with the Poisson bracket

$$
-\mathrm{d} F\lrcorner \mathrm{d} H\lrcorner \Psi=\{F, H\}=\frac{1}{2} \sum \int_{\Omega} D_{k+l}\left(E^{k}(f) D E^{l}(h)-E^{k}(h) D E^{l}(f)\right) .
$$

4. Dynamics on a boundary: an example. Let us now consider a simple Hamiltonian system in order to obtain boundary equations with the help of the new brackets. The nonlinear Schrödinger equation

$$
i \dot{\psi}=-\psi^{\prime \prime}+2 k \psi|\psi|^{2},
$$

can be treated $[\mathrm{LR}]$ as generated by the Hamiltonian

$$
H=\frac{1}{2} \int(\mathcal{H}+\overline{\mathcal{H}}) d x
$$

where

$$
\mathcal{H}=r^{\prime} q^{\prime}+k r^{2} q^{2}, \quad \overline{\mathcal{H}}=\bar{r}^{\prime} \bar{q}^{\prime}+k \bar{r}^{2} \bar{q}^{2},
$$

and Poisson brackets are

$$
\{q(x), r(y)\}=-2 i \delta(x, y), \quad\{\bar{q}(x), \bar{r}(y)\}=2 i \delta(x, y) .
$$

To return to the standard form of this equation we should put reality conditions

$$
\psi=q=\bar{r}, \quad \bar{\psi}=r=\bar{q} .
$$

Let us calculate the full variational derivatives

$$
\frac{\delta H}{\delta q}=k r^{2} q-\frac{1}{2} r^{\prime \prime}-\frac{1}{2} \theta^{\prime} r^{\prime}, \quad \frac{\delta H}{\delta r}=k r q^{2}-\frac{1}{2} q^{\prime \prime}-\frac{1}{2} \theta^{\prime} q^{\prime},
$$

analogous formulas take place for the bar variables.

The natural boundary condition arises if we put $\delta$-function contribution on the boundary to zero by taking

$$
q^{\prime}=r^{\prime}=\bar{q}^{\prime}=\bar{r}^{\prime}=0 .
$$

By considering Poisson brackets for integrals of the total spatial derivatives of canonical variables $\phi_{A}=(q, r)$ with the Hamiltonian we expect to obtain dynamical equations on the boundary in functional form

$$
\frac{d}{d t} \int \phi_{A}{ }^{\prime} d x=\left\{\int \phi_{A}{ }^{\prime} d x, H\right\}=\int D\left(\frac{1}{2} \sum_{B} I_{A B} \frac{\partial \mathcal{H}}{\partial \phi_{B}}\right) d x .
$$


Using Newton-Leibniz formula we get

$$
\left.\dot{\phi_{A}}\right|_{1} ^{2}=\left.\frac{1}{2} \sum_{B} I_{A B} \frac{\partial \mathcal{H}}{\partial \phi_{B}}\right|_{1} ^{2} .
$$

This is different from the standard (bulk) equations

$$
\dot{\phi}_{A}=\frac{1}{2} \sum_{B} I_{A B} E_{B}^{0}(\mathcal{H}) .
$$

For the given Hamiltonian the formal equations for boundary values are

$$
\dot{q}_{b}=-2 i k r_{b} q_{b}^{2}, \quad \dot{r}_{b}=2 i k r_{b}^{2} q_{b}
$$

if we assume independent behaviour at the ends. It is remarkable that the boundary equations are different from the bulk ones despite the boundary condition. These equations can be easily integrated and give elementary oscillations at the ends

$$
\psi_{b}(t)=\psi_{b}(0) \exp \left(-2 i k\left|\psi_{b}(0)\right|^{2} t\right),
$$

where the initial value of $\psi$ determines both amplitude and frequency of the oscillator.

In this case the dynamics of boundary values is separated from the bulk dynamics. Of course, this situation is not general.

Let us mention that our boundary condition is compatible with the dynamics, for example,

$$
\left.\frac{d}{d t} q^{\prime}\right|_{1} ^{2}=\left\{\int q^{\prime \prime}, H\right\}=-\left.2 i k r^{\prime}\right|_{1} ^{2}=0
$$

5. Discussion. There are not so many publications on problems where divergences play a nontrivial role in Hamiltonian formalism of field theory. After the classical paper by Regge and Teitelboim [RT] we can recommend the work by Jezierski and Kijowski $[\mathrm{JK}]$ (see also book $[\mathrm{KT}]$ ) where the main criterion is also the disappearance of surface terms in the first variation of the Hamiltonian. Such functionals are called admissible or differentiable, but to be convinced in the full consistency of the formalism it is necessary to check the two points:

1) the space of admissible local functionals should be closed under the Poisson bracket;

2) the Jacobi identity should be fulfilled for arbitrary admissible functionals;

and this check is not explicitly demonstrated in the cited works. For infinite domain the first requirement was studied by Brown and Henneaux [BH]. In the finite domain case the important contribution was made by Lewis, Marsden, Montgomery and Ratiu [LMMR] who showed that the standard bracket did not fulfil the Jacobi identity and proposed to modify it by adding some surface terms. Unfortunately these authors do not consider explicitly the first requirement. We hope that our results could serve as a generalization of their ansatz and could be useful in dealing with interesting problems of field theory.

6. Acknowledgements. The author is most grateful to Professor J. Kijowski for the invitation and to the Stefan Banach Center for hospitality and support.

Discussions with M. Asorey, J. Jezierski, I. Kanatchikov, J. Kijowski, J. Louko, J. Nester and other participants are gratefully acknowledged. 


\section{References}

[Ald] S. J. Aldersley, Higher Eulerian operators and some of their applications, J. Math. Phys. 20 (1979), 522-531.

[And76] I. M. Anderson, Mathematical foundations of the Einstein field equations, Ph. D. thesis, Univ. of Arizona, 1976.

[And78] I. M. Anderson, Tensorial Euler-Lagrange expressions and conservation laws, Aequationes Math. 17 (1978), 255-291.

[And92] I. M. Anderson, Introduction to the variational bicomplex, in: Mathematical aspects of classical field theory, M. J. Gotay, J. E. Marsden and V. Moncrief (eds.), Contemp. Math. 132, AMS, Providence, 1992.

[Arn] V. I. Arnol'd, Mathematical methods of classical mechanics, Nauka, Moscow, 1974 (in Russian).

[ADM] R. Arnowitt, S. Deser and C. W. Misner, Consistency of the canonical reduction of General Relativity, J. Math. Phys. 1 (1960), 434-439.

[BH] J.D. Brown and M. Henneaux, On the Poisson brackets of differential generators in classical field theory, J. Math. Phys. 27 (1986), 489-491.

[Dorf] I. Dorfman, Dirac Structures and Integrability of Nonlinear Evolution Equations, John Wiley and Sons, New York, 1993.

[GD] I. M. Gel' fand and L. A. Dickey, Asymptotics of Sturm-Liouville equation resolvent and algebra of Korteweg-de Vries equation, Uspekhi Mat. Nauk 30 (1975), 67-100 (in Russian).

[JK] J. Jezierski and J. Kijowski, The localization of energy in gauge field theories and in linear gravitation, Gen. Relativity Gravitation 22 (1990), 1283-1307.

[KT] J. Kijowski and W. M. Tulczyjew, A symplectic framework for field theories, Lecture Notes in Phys. 107, Springer, New York, 1979.

[KMGZ] M. D. Kruskal, R. M. Miura, C. S. Gardner and N. J. Zabusky, Korteweg-de Vries equation and generalizations. V. Uniqueness and nonexistence of polynomial conservation laws, J. Math. Phys. 11 (1970), 952-960.

[LMMR] D. Lewis, J. Marsden, R. Montgomery and T. Ratiu, The Hamiltonian structure for dynamic free boundary problems, Phys. D 18 (1986), 391-404.

[LR] A. N. Leznov, A. V. Razumov, The canonical symmetry for integrable systems, J. Math. Phys. 35 (1994), 1738-1754.

[Nij] A. Nijenhuis, Jacobi-type identities for bilinear differential concomitants of certain tensor fields. I, Indag. Math. 17 (1955), 390-397.

[Olv84] P. J. Olver, Hamiltonian perturbation theory and water waves, in: Fluids and Plasmas: Geometry and Dynamics, J. E. Marsden (ed.), Contemp. Math. 28, AMS, Providence, 1984.

[Olv86] P. J. Olver, Applications of Lie Groups to Differential Equations, Graduate Texts in Mathematics, Springer-Verlag, New York, 1986.

[RT] T. Regge and C. Teitelboim, Role of surface integrals in Hamiltonian formalism of General Relativity, Ann. Physics 88 (1974), 286-318.

[Sol85] V. O. Soloviev, Algebra of asymptotic Poincaré group generators in General Relativity, Teoret. Mat. Fiz. 65 (1985), 400-415 (in Russian).

[Sol92] V. O. Soloviev, How canonical are Ashtekar's variables?, Phys. Lett. B 292 (1992), 30-34. 
[Sol93] V. O. Soloviev, Boundary values as Hamiltonian variables. I. New Poisson brackets, J. Math. Phys. 34 (1993), 5747-5769.

[Sol94] V. O. Soloviev, Boundary values as Hamiltonian variables. II. Graded structures, q-alg/9501017, Preprint IHEP 94-145, Protvino, 1994. 